\title{
Minimizing bugs in cognitive neuroscience programming
}

\author{
Vadim Axelrod ${ }^{1,2 *}$ \\ ${ }^{1}$ The Gonda Multidisciplinary Brain Research Center, Bar Ilan University, Ramat Gan, Israel \\ 2 Institute of Cognitive Neuroscience, University College London, London, UK \\ *Correspondence: vadim.axelrod@gmail.com \\ Edited by: \\ Michael J. Tarr, Carnegie Mellon University, USA \\ Reviewed by: \\ Pierre Bellec, University of Montreal, Canada \\ John A. Pyles, Carnegie Mellon University, USA
}

Keywords: programming, bugs, code quality, minimizing errors, code testing

\section{INTRODUCTION}

Rigorous data analysis is a cornerstone of empirical scientific research. In recent years, much attention in the field of cognitive neuroscience has been paid to the issue of correct statistical procedures (e.g., Kriegeskorte et al., 2009; Nieuwenhuis et al., 2011; Kilner, 2013). However, an additional essential aspect of data analysis, which has attracted relatively little attention, is the errors (bugs) in custom data analysis programming code. Whereas, in its broad definition a bug can be any type of an error, here I refer to it as a problem in the code that does not lead to a failure (error or crash) during execution; that is, code that contains a bug completes its execution properly, but the output result is incorrect. Notably, if an erroneous output result consists of reasonable values, then the programmer might have no indication that something went wrong. It is impossible to estimate how many published studies contain results with bugs; however, given the ubiquity of bugs in non-scientific (industrial) software (Zhivich and Cunningham, 2009), it is plausible that academic code is no exception. Furthermore, the quality of custom code in cognitive neuroscience field in particular, is probably even worse than in the industry because (a) code in the field of cognitive neuroscience is usually written by people with only basic programming training-after all, they are brain researchers and not software engineers; (b) the code most often is programmed by a single researcher, without any code peer-review procedure (Wiegers, 2002); and (c) the custom code is usually used by a single or few lab members only. This last point is critical, because when the code is used by many, as is the case with large open-source projects like SPM (http://www.fil.ion.ucl.ac.uk/spm/), FieldTrip (http://fieldtrip.fcdonders.nl/), EEGLab (http://sccn.ucsd.edu/eeglab/) or PyMVPA (http://www.pymvpa.org/), the likelihood is low that a serious bug would remain unnoticed for a long time. The goal of this paper is to give practical advice to cognitive neuroscientists on how to minimize bugs in their custom data analysis code. The advice is illustrated using MATLAB schematic samples; executable code examples can be found in the Supplementary Materials.

\section{ADVICE 1: THE DANGERS OF IRRELEVANT DATA}

Consider a case of an fMRI data analysis, where a researcher needs to calculate an average level of activation (e.g., z-score) for the predefined regions of interest (ROIs). The code logic goes as follows: (1) iterate over ROI files, and get the coordinates for each ROI; (2) for each voxel in the ROI, retrieve its $\mathrm{Z}$-score value; and (3) average values across ROIs' voxels, and store all the results in a table. Please see the code sample 1 in Box 1.

The problem with this code is that the roi_zscore_vector variable is not cleaned up between ROI loop cycles. So, if, for example, ROI No. 3 consists of 127 voxels and ROI No. 4 consists of only 7, then the mean result for ROI No. 4 will be based on 7 correct $\mathrm{z}$-score values and 120 incorrect $\mathrm{z}$ score values from the previous loop cycle. The easy way to fix the bug is to clean up the roi_zscore_vector variable between loop cycles (e.g., roi_zscore_vector=[];). It worth noting that a more secure and efficient way to write the code would be to retrieve all ROI's z-score values at once, instead of iterating over the single voxels ("vectorized" approach). Please see the code sample 2 in Box 1.

Bugs caused by irrelevant data might be as simple as the use of an incorrect index in the loop, misuse of the variable or the use of incorrect configuration file. These bugs usually occur as a result of an inaccuracy or momentary attentional lapse. There is no complete remedy for this problem, but adopting good coding practices (see Advice 4: Bugs and Code Quality) decreases the likelihood of such errors.

\section{ADVICE 2: TEST YOUR CODE!}

A fact not immediately appreciated by inexperienced programmers is that regardless of how hard one tries, it is difficult not to make errors during programming. The important thing is to catch these errors in time, so they do not remain unnoticed (i.e., become bugs). To this extent, test procedures substantially improve code quality. Below are several recommendations:

\section{USE A DEBUGGER TO VALIDATE WHETHER YOUR CODE WORKS AS EXPECTED}

Once the code is written, debug it first before fully executing it from beginning to end. That is, set a breakpoint(s) within the critical parts of the code; then, by using debugger step functionality, validate line by line that the flow of the program is as expected and the intermediate calculations are correct. In general, no quality code can be produced without using a debugger. 


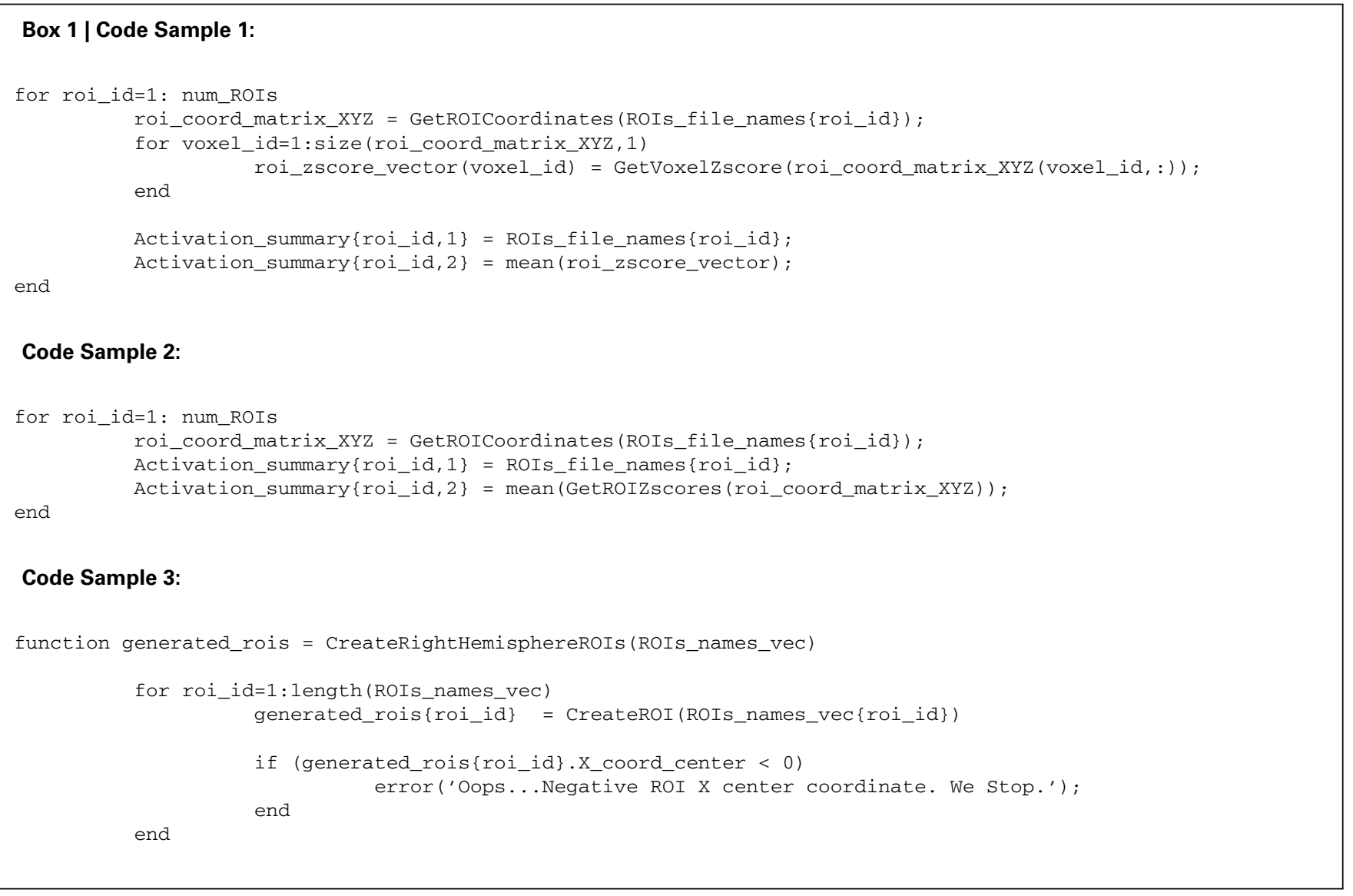

\section{KEEP TRACK AND VALIDATE INTERMEDIATE RESULTS}

Keep track of intermediate calculation results by outputting them to command window, and preferably, also by saving them in a log file(s) or as graphical figures. This approach allows you: (a) to establish whether something went wrong during execution, and (b) to compare intermediate results between code executions.

\section{COMPARE AUTOMATIC RESULTS WITH THOSE CREATED MANUALLY}

A useful way to validate whether the results of custom code are correct is to compare them with the results generated manually. For example, if your code automatically converts z-score fMRI $t$-contrast images to binary masks, it is worth comparing the automatically generated masks with the masks created manually (e.g., using SPM's imcalc).

\section{USE SIMULATED (SURROGATE) DATA TO TEST THE CODE}

The use of a simulated dataset as opposed to real data is the only unbiased way to ensure that the code works as expected and does not contain bugs. The simulated dataset can help to ensure that the code finds the effect of interest when the data indeed contains it, and also that no effect is found when the data is a random noise. PyMVPA provides an excellent example of surrogate data use.

\section{ADVICE 3: PROTECTIVE (PREVENTIVE) PROGRAMMING}

The essence of the protective programming approach is that the program validates its intermediate results as it runs, and alerts the programmer in real time in the event of a problem. Consider, for example, the code that creates a collection of ROI masks for the right hemisphere according to the Montreal Neurological Institute (MNI) coordinate system. Accordingly, the $\mathrm{X}$ coordinate of all ROIs should be positive. Please see the code sample 3 in Box 1.

If for some reason the center coordinate of the created ROI was negative, then the code would just stop. Critically, this defensive check prevents further propagation of the error (see also assert function).
In general, it is recommended that the mission-critical parts of the code, and possibly also input and output function parameters be validated. Remember, getting a fatal error during code execution is preferable to searching for elusive bugs in code that completes execution without errors.

\section{ADVICE 4: BUGS AND CODE QUALITY}

Novice programmers tend to pay little attention to how they organize the code. This is a bad practice, because when the code is messy and illegible, programmers have a higher propensity to make errors and confusion.

\section{USE MEANINGFUL FUNCTION AND VARIABLE NAMES}

Good name convention helps make sense of the logic of the code, and also serves the role of documentation. For example, when functions are named CreateBinaryMask or FindPeakActivationWithinROI, it is immediately clear what they are doing. The variables should also be given meaningful names like voxelID and subjectID, instead 
of $i$ or $j$. The use of proper name convention for variables also decreases the chances of irrelevant data use (see Advice 1: The Dangers of Irrelevant Data). That is, the use of meaningful names makes it more difficult to get confused between variables because (a) they are less similar visually (visual saliency), and (b) they refer to specific concepts (e.g., voxels, subjects).

\section{DO NOT DUPLICATE ("COPY-PASTE") YOUR CODE}

The main problem with this practice is that the code becomes unmanageable. That is, if the logic that was duplicated ever needs to be changed, locating and changing all of the duplicated instances is a process highly prone to error. The solution is to extract the code that needs duplication to a separate function (see next item).

\section{USE A MODULAR APPROACH}

The best way to maintain and reuse the code is to adopt a modular approach. According to this method, separate business logic should be implemented in separate functions (e.g., see code samples in Box 1). The modular code is more readable because top-level flow is not cluttered by the implementation. Critically, the nonmodular code contains many variables in its scope, most of which are, at any given stage of execution, completely irrelevant. This, in turn, increases the likelihood of misuse of these variables (see Advice 1: The Dangers of Irrelevant Data).

\section{AVOID HARD-CODED VALUES}

The business logic code should not contain hard-coded values unless those values are likely to never change (e.g., $60 \mathrm{~s}$ in minute). Initial parameters that might be changed should be either passed as parameters to the function or defined at the top of the code in a transparent way. A good practice is also to save the initial parameters for each execution, so that it would be possible to reproduce the results.

\section{DO NOT LEAVE UNSUPPORTED INITIAL PARAMETERS (OR ANY DEFINITIONS) IN THE CODE}

During code development, some initial parameters might become obsolete. For example, at the beginning, the data analysis was conducted for a single ROI that was defined as:
INITIAL_PARAMS.ROI = 'V1.nii';

Then, the support for multiple ROIs was added.

INITIAL_PARAMS.ROIS_arr $=\ldots$ \{'V1.nii', 'V2.nii', 'V3.nii'\};

If INITIAL_PARAMS.ROI is no longer supported, I strongly recommend deleting or at least commenting on its definition. The main reason is that, if, by mistake, there is still a code that uses INITIAL_PARAMS.ROI (see Advice 1: The Dangers of Irrelevant Data), then the results of code execution would likely be wrong. By removing the obsolete definition, we ensure that no occasional use is made; if it is, then the code will throw an error (see Advice 3: Protective (preventive) Programming).

\section{MAKE YOUR CODE AS UNDERSTANDABLE AS POSSIBLE}

Logic of some code might be so complicated that not only others, but even the programmer himself after some time, might struggle to understand it. Following the steps suggested above will make the code more readable and understandable. In addition, adding comments, especially to the most critical or non-trivial parts of the code, might prevent future frustration.

\section{ADOPT PEER-REVIEW PROCEDURE}

Peer-review is the procedure by which you show and explain the code you wrote to your colleague(s). This is an extremely helpful procedure, not only because your colleagues might give you valuable advice, but also because explaining the code to someone else makes you look at your code more critically.

\section{ADVICE 5: CONSULT LANGUAGE DOCUMENTATION WHEN NEEDED}

Programming modern language, such as MATLAB or Python, is easy and intuitive. However, there are some cases when intuition is not sufficient. Consider a case when MATLAB is used in fMRI Multivariate Pattern Analysis (MVPA). A widely used preprocessing practice is to standardize a time course for each voxel (e.g., Misaki et al., 2010). To achieve this, the zscore function is called with a 2-D matrix of "voxels $x$ data-volumes" parameter (e.g., Reddy et al., 2010; Lee et al., 2011; Axelrod et al., 2014). Critically, without consulting the documentation, one cannot be sure along which dimension (voxels or data-volumes) the zscore function makes the standardization. As a result, one can end up with an absolutely wrong output result that might look absolutely correct (i.e., correct dimensions). The solution in such cases is not to trust your intuition but to consult the documentation.

\section{ADVICE 6: TAKE RESPONSIBILITY FOR THE CODE YOU USE}

It is common for researchers or students to use code written by someone else in their own or another lab. There are two potential caveats here. First, the person who "inherited" the code might not fully understand how the code should be used. Second, the original code may have been written for another purpose and therefore might not work correctly in the new situation. All this might lead to dangerous bugs. The only solution is to fully understand the code you are using, regardless of whether you wrote it or not.

\section{ADVICE 7: MAKE YOUR CODE PUBLIC, IF POSSIBLE}

If you are brave enough, you might want to publish the custom code you used for your publication (e.g., Hermes et al., 2014). This permits other researchers not only to validate your code, but also to replicate your analyses (Brunet et al., 2014). Admittedly, publishing code is of limited use when the data it uses are not published, which is the case with most neuroimaging studies to date.

\section{ADVICE 8: USE SOURCE MANAGEMENT SYSTEMS}

The use of source management systems (e.g., https://github.com/) makes the management of code versions easy. Version control systems permit researchers to document their changes and compare between versions; critically, they permits a researcher to establish exactly when a certain change (or bug) was introduced in the code.

\section{CONCLUDING REMARKS}

This paper provided practical advice on minimizing bugs in cognitive neuroscience 
Table 1 | Summary of actions that can be taken to improve the code.

\begin{tabular}{ll}
\hline Type of advice & Actions to take \\
\hline Test your code & Use debugger to test your code \\
& Keep track and validate intermediate results (e.g., save log file, image figures) \\
& Validate the output of custom code using the manually created results \\
& Check your code with simulated (surrogate) data
\end{tabular}

Protective (preventive) programming

Irrelevant data and code quality
Make your code to check the intermediate output results as it runs

Make sure the variables do not contain data from previous operations (e.g., loop cycle)

Extract specialized business logic to separate functions (modular approach)

Do not duplicate code

Use meaningful names for functions and variables

Avoid hard-coded values within the code

Do not leave unsupported variables (e.g., initial parameters) in the code

Add comments to the most critical and non-trivial parts of the code

Adopt peer-review procedure

Consult documentation

Do not trust your intuition. Make sure that the functions are called according to documentation

Take responsibility for the code you use

Do not use the code without understanding it

Publish your code, if possible

Make your code available to other researchers

Use source management systems

Source systems permit to manage and document your code easily data analysis programming (see, summary in Table 1). Probably the most important take-home message is not to assume that the code you write is bug-free; that is, even a small piece of relatively simple code may contain a bug that could lead to incorrect results. Therefore, it is important not to be complacent and to take steps that can prevent such failures.

\section{ACKNOWLEDGMENTS}

I thank Benjamin de Haas, Samuel Schwarzkopf and two reviewers for valuable suggestions.

\section{SUPPLEMENTARY MATERIAL}

The Supplementary Material for this article can be found online at: http:// www.frontiersin.org/journal/10.3389/fpsyg 2014.01435/full

\section{REFERENCES}

Axelrod, V., Bar, M., Rees, G., and Yovel, G. (2014). Neural correlates of subliminal language processing. Cereb. Cortex. doi: 10.1093/cercor/bhu022. [Epub ahead of print].

Brunet, N., Vinck, M., Bosman, C. A., Singer, W., and Fries, P. (2014). Gamma or no gamma, that is the question. Trends Cogn. Sci. 18, 507-509. doi: 10.1016/j.tics.2014.08.006
Hermes, D., Miller, K., Wandell, B., and Winawer, J. (2014). Stimulus dependence of gamma oscillations in human visual cortex. Cereb. Cortex bhu091. doi: 10.1093/cercor/bhu091. [Epub ahead of print].

Kilner, J. (2013). Bias in a common EEG and MEG statistical analysis and how to avoid it. Clin. neurophysiol. 124, 2062. doi 10.1016/j.clinph.2013.03.024

Kriegeskorte, N., Simmons, W. K., Bellgowan, P. S. F., and Baker, C. I. (2009). Circular analysis in systems neuroscience: the dangers of double dipping. Nat. Neurosci. 12, 535-540. doi: 10.1038/nn. 2303

Lee, Y.-S., Janata, P., Frost, C., Hanke, M., and Granger, R. (2011). Investigation of melodic contour processing in the brain using multivariate pattern-based fMRI. Neuroimage 57, 293-300. doi: 10.1016/j.neuroimage.2011. 02.006

Misaki, M., Kim, Y., Bandettini, P. A., and Kriegeskorte, N. (2010). Comparison of multivariate classifiers and response normalizations for pattern-information fMRI. Neuroimage 53, 103-118. doi: 10.1016/j.neuroimage.2010. 05.051

Nieuwenhuis, S., Forstmann, B. U., and Wagenmakers, E.-J. (2011). Erroneous analyses of interactions in neuroscience: a problem of significance. Nat. Neurosci. 14, 1105-1107. doi: 10.1038/nn.2886

Reddy, L., Tsuchiya, N., and Serre, T. (2010). Reading the mind's eye: decoding category information during mental imagery. Neuroimage
50, 818-825. doi: 10.1016/j.neuroimage.2009. 11.084

Wiegers, K. E. (2002). Peer Reviews in Software: a Practical Guide. Available online at: http:// www.informit.com/store/peer-reviews-in-softwarea-practical-guide-9780201734850

Zhivich, M., and Cunningham, R. K. (2009). The real cost of software errors. Secur. Priv. IEEE 7, 87-90. doi: $10.1109 / \mathrm{msp}$. 2009.56

Conflict of Interest Statement: The author declares that the research was conducted in the absence of any commercial or financial relationships that could be construed as a potential conflict of interest.

Received: 31 October 2014; accepted: 24 November 2014; published online: 17 December 2014.

Citation: Axelrod V (2014) Minimizing bugs in cognitive neuroscience programming. Front. Psychol. 5:1435 doi: 10.3389/fpsyg.2014.01435

This article was submitted to Perception Science, a section of the journal Frontiers in Psychology.

Copyright (c) 2014 Axelrod. This is an open-access article distributed under the terms of the Creative Commons Attribution License (CC BY). The use, distribution or reproduction in other forums is permitted, provided the original author(s) or licensor are credited and that the original publication in this journal is cited, in accordance with accepted academic practice. No use, distribution or reproduction is permitted which does not comply with these terms. 